\title{
Adenoma recurrence after endoscopic mucosal resection: propensity score analysis of old and new colonoscopes and Sydney recurrence tool implementation
}

\section{(ㄷ)(우)}

\author{
Authors \\ Daniela Guerrero Vinsard1,3, Pujan Kandel1, Lady Katherine Mejia Perez¹, Russell L. Bingham', Ryan J. Lennon², \\ Timothy A. Woodward ${ }^{1}$, Victoria Gomez ${ }^{1}$, Massimo Raimondo ${ }^{1}$, Ernest P. Bouras ${ }^{1}$, Michael B. Wallace ${ }^{1}$
}

Institutions

1 Division of Gastroenterology and Hepatology, Mayo Clinic, Jacksonville, Florida, United States

2 Division of Biomedical Statistics and Informatics, Mayo Clinic, Rochester, Minnesota, United States

3 Division of Internal Medicine, University of Connecticut Health Center, Farmington, Connecticut, United States

submitted 14.6.2017

accepted after revision 25.10.2017

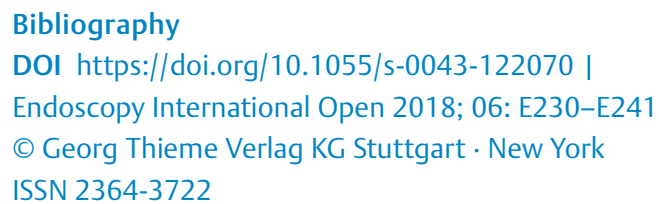

Corresponding author

Michael B. Wallace, MD, MPH, Division of Gastroenterology and Hepatology, Mayo Clinic, 4500 San Pablo Road, Jacksonville, FL 32224

Fax: +1-904-953-6225)

wallace.michael@mayo.edu

\section{ABSTRACT}

Background and study aims Risk factors for colorectal adenoma recurrence after endoscopic mucosal resection (EMR) have been well documented. We assessed the effica- cy of the newer 190 colonoscope versus the standard 180 colonoscope for complete resection of lateral spreading lesions.

Patients and methods A single-center, retrospective study of patients who underwent EMR with Olympus 180 or 190 colonoscopes from January 1, 2010 to September 30,2016 . We included patients with lesions $\geq 20 \mathrm{~mm}$ and surveillance colonoscopy (SC1) after index EMR. A propensity score approach with inverse probability weighting was used to control for potential confounders. A secondary aim was to identify risk factors for recurrence and assess the applicability of the Sydney EMR recurrence tool (SERT) by grading each lesion of our cohort and analyzing associations with recurrence.

Results Two hundred ninety-one lesions met inclusion criteria for the study. Odds ratio (OR) for recurrence with the 190 colonoscope was $1.06(P=.85)$. Adenoma size $(P$ $=.02$ ) and use of argon plasma coagulation (APC; $P<.001)$ were risk factors for recurrence. Lesions with SERT scores $>0$ had a higher recurrence risk during follow-up (32\% vs $21 \%$; OR $1.71 ; P=.05)$. Lesions with SERT scores $=0$ reached a plateau for recurrence at 12 and 18 months in Kaplan-Meier curves.

Conclusions The use of 190 colonoscopes did not measurably affect adenoma recurrence at SC1. Recurrence was associated with adenoma size, complementary APC for resection, and SERT scores $>0$. Lesions with SERT scores $=0$ that remain negative for recurrence at 18 months may return to routine surveillance.

\section{Introduction}

The American Society for Gastrointestinal Endoscopy (ASGE) Technology Committee defined the quality of endoscopic visualization as a function of video resolution and magnification [1]. High definition (HD), high power of magnification, and chromoendoscopy have been implemented as potential imaging enhancers during colonoscopy with the purpose of increasing the detection, pit pattern characterization, and targe- ted treatment of colonic lesions while decreasing the miss rate and unnecessary pathologic evaluation of non-neoplastic polyps. When large $(>1 \mathrm{~cm})$, laterally spreading lesions $(\mathrm{LSL})$ are detected; endoscopic mucosal resection (EMR) can be performed as a minimally invasive technique with high success rates and lower morbidity, mortality, and cost when compared to surgery [2].

At the beginning of every EMR procedure a detailed visualization to establish the margins, particularly of flat lesions, is 
crucial to ensure a complete resection and minimize the risk of residual or recurrent adenoma (RRA). A recent study by Desomer et al [3] demonstrated that HD narrow-band imaging (HD-NBI) detects RRA with improved accuracy compared to white light. The newly available second generation 190 colonoscopes (190-NBI; see description below) provides at least 2-fold brighter HD images, with increased contrast and decreased halation compared to the previous version of colonoscopes [4]. The 190 colonoscope further allows polyp examination in standard and near-focus modes granting a true optical in-focus zoom [5]. Additionally, it has twice the viewable distance of 180 colonoscopes. An important question raised in a recent editorial to the Desomer paper by Cohen [6] is whether this advanced optical system reduces RRA by improving the initial visualization of affected tissue and subsequently improves complete endoscopic resection of LSLs. This question was the impetus for our study.

Intralesional and extralesional risk factors for adenoma recurrence after EMR have been well described in multiple studies [2, 7-11]. A more recent prospective multicenter study by Tate et al [12] proposed a scoring model for stratification of recurrence risk after EMR. The Sydney EMR recurrence tool (SERT) is a 0 to 4 point scale that grades a lesion based on a size of $40 \mathrm{~mm}$ or larger (2 points), presence of intraprocedural bleeding (IPB; 1 point), and high-grade dysplasia (HGD) in histopathology (1 point) [12]. The authors concluded that a score of 0 entails a low risk for recurrence at 6 months; therefore, these patients could safely undergo first surveillance colonoscopy (SC1) at 18 months. If clinically applicable, stratification of recurrence risk may considerably reduce the costs of colon cancer surveillance and bypass unnecessary histopathologic evaluation.

\section{Patients and methods}

The Mayo Clinic Institutional Review Board approved the study. The primary aim of this study was to assess the efficacy of the newer CF-HQ190L/I colonoscopes versus the standard CF$\mathrm{H} 180 \mathrm{AL} / \mathrm{I}$ colonoscopes in the complete resection of LSL $20 \mathrm{~mm}$ or larger as demonstrated by a reduction in the rate of RRA at the EMR site during SC1. A secondary aim was to identify risk factors for RRA in our study population and assess the clinical applicability of the SERT score for further surveillance recommendations.

\section{Patients}

Consecutive patients who underwent EMR of colorectal polyps from January 1, 2010 to September 30, 2016 were extracted from the Mayo Clinic, Jacksonville, Florida, ProVation MD system and their electronic medical records were retrospectively reviewed. Of 836 resected lesions, we included 291 in patients who met the inclusion criteria for the study: LSL $20 \mathrm{~mm}$ or larger and at least 1 surveillance colonoscopy (SC) after index EMR for evaluation of RRA. If the patient had more than $1 \mathrm{LSL} 20 \mathrm{~mm}$ or larger treated with EMR, every lesion was included in the study. Other inclusion and exclusion criteria are described in Box.
INCLUSION AND EXCLUSION CRITERIA FOR STUDY

ELIGIBILITY

Inclusion criteria

- Age $\geq 18$ years

- Colonoscopy done with either Olympus 180 or 190 series colonoscopes

- Lateral spreading lesions $\geq 20 \mathrm{~mm}$

- Lesions adequately/partially lifted prior to EMR

- Lesions completely resected and retrieved according to endoscopist's assessment

- Polyps with or without prior therapy

\section{Exclusion criteria}

- Patients who failed to have a surveillance colonoscopy after index EMR done in our facility

- Lesions resected en bloc (excluded only for IPW analysis)

EMR, endoscopic mucosal resection; IPW, inverse probability weighting

Patients who failed to come back to our facility for SC after index EMR were excluded from the study. All collected data were filtered for inclusion criteria as shown in > Fig. 1.

\section{Procedures and endoscopy equipment}

The procedure was performed by 1 of the 5 EMR specialists in our gastroenterology department. HD endoscopy with Olympus CF-H180AL/I and Olympus CF-HQ190L/I colonoscopes (Olympus America, Center Valley, Pennsylvania), and with EVIS EXERA II CV-180 and EVIS EXERA III CV-190 (Olympus Medical Systems, Tokyo, Japan) video processors were performed to characterize all lesions.

Olympus 190 colonoscopes were introduced in our practice at the end of 2013. From 2010 to early 2013, every patient included in our study underwent EMR with the 180 series. Starting in 2014, patients underwent EMR with either the 180 or 190 systems depending on the colonoscope availability at the time of the procedure. This assignment was therefore not a result of operator preference or patient characteristics.

White light imaging and HD-NBI were performed to inspect the mucosa. If the 190 scope was employed, zoom magnification (near focus or NF) of the lesion was used at the discretion of the endoscopist. Polyp size, anatomic site, and macroscopic morphology using the Paris classification were recorded [13]. Polyps were removed using the inject, lift, and cut method [14]. If removal was incomplete, snare tip soft coagulation, argon plasma coagulation (APC), or hot forceps avulsion were used to achieve complete removal of lesions. All polyps were sent for histopathologic analysis, and specialized gastrointestinal pathologists performed histologic examination.

\section{Surveillance colonoscopy: defining recurrence}

Repeat colonoscopy in 6 months was recommended for surveillance. SC and EMR scar assessment were done by an EMR specialist, and biopsy or snare resection of the scar were per- 
Lesions treated with EMR from 2010-2016 ( $n=836)$

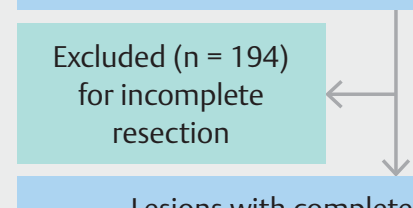

Lesions with complete resection $(n=642)$

Excluded $(n=49)$

due to different

system used

(e. g. 160 scope)

180 or 190 system used $(n=593)$

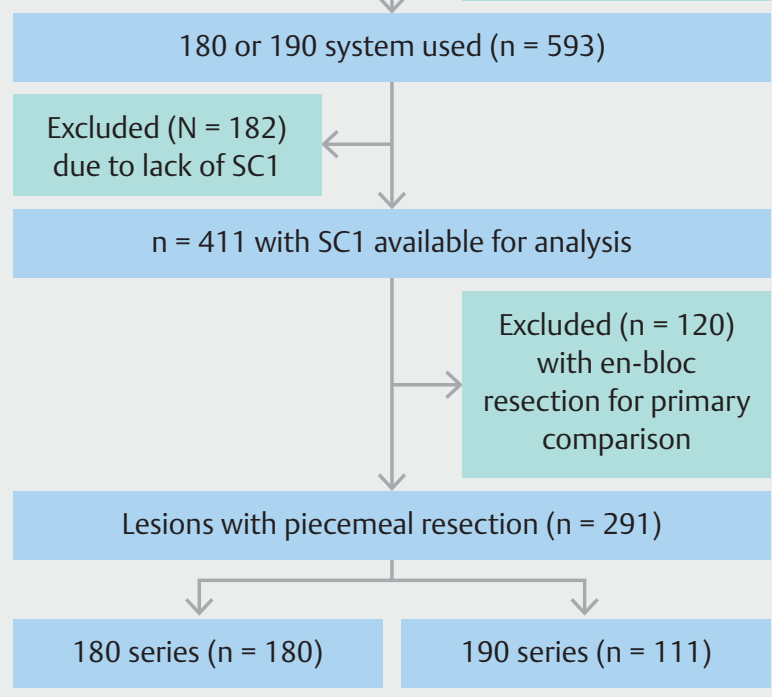

Fig. 1 Study flow for data collection.

formed if there was any suspicion of recurrence. The post-EMR scar was assessed with HD-NBI of the lesion with either 180 or 190 colonoscopes depending on availability. If there was high confidence of no residual/recurrent neoplasia, it was assumed to be negative. We have previously confirmed that such inspection has a high negative predictive value in a recent study by Kandel et al in our center. In all other cases biopsy samples were taken and histology served as the reference standard for recurrence.

\section{Statistical analysis}

Categorical variables were reported as numbers and percentages with group differences compared using the $X^{2}$ test. Continuous variables were reported as the mean (SD) and interquartile range (IQR; first and third quartiles), and differences between 2 groups were evaluated using a $t$ test. Due to substantial confounding between the colonoscope used and the type of resection (piecemeal vs en bloc), every procedure with en bloc resection was excluded from the comparative analysis. Logistic regression was used to estimate the odds ratio (OR) and Cls for risk factors and associations with recurrence.
A propensity score was developed using logistic regression to model the likelihood of the scope used based on patient and procedural characteristics. The variables used to construct the propensity score were age, sex, prior treatment of the lesion, polyp size, EMR location, Paris classification, endoscopist, lesion adequately lifted, IPB, use of APC, and initial pathology. Additionally, interactions between age and sex and initial pathology and prior treatment were included to improve covariate balance. Inverse probability weights (IPWs) were assigned to each patient according to the reciprocal of the estimated probability of being in their observed group. Weights were then normalized so that the weights within each group totaled the group sample size. On the weighted data, standardized differences were calculated to assess imbalance for continuous variables, differences in proportions were used for binary variables. A difference greater than 0.10 was considered a sign of imbalance.

The SERT score was calculated in all patients with piecemeal resection and a score above 0 was considered high-risk for recurrence. Sensitivity and specificity measures for the association between a SERT score above 0 and the incidence of any recurrence were calculated. ORs for recurrence at any point in follow-up were calculated using logistic regression. A multiple logistic regression model was used to estimate adjusted ORs for the 3 components of the SERT score. Recurrence was also estimated using Kaplan-Meier method, recognizing that this approach has limitations, given that the time of recurrence is not continuously monitored and is dependent on the timing of the follow-up colonoscopy.

Analyses were 2-tailed, and the threshold for statistical significance was $P<.05$. Analyses were conducted using $R$ Software (R Foundation for Statistical Computing, Vienna, Austria).

\section{Results}

\section{Patient population and 180 versus 190 colonoscopes}

A total of 378 patients with 411 resected lesions met the initial inclusion criteria (en bloc and piecemeal resections). The 180 colonoscope was used in $72 \%$ of resections $(n=296)$ and the 190 colonoscope was used in $28 \%(n=115)$. Mean age was 67 years $( \pm 10)$ with a median polyp size of $30 \mathrm{~mm}(20-140 \mathrm{~mm})$. Median time to SC1 was 5 months (IQR 15-30 weeks). Some differences in baseline characteristics between the 2 groups included age, prior treatment of polyp, initial histopathology, use of APC, resection type, year of procedure, and endoscopist performing EMR, which were adjusted with IPW before final analysis. The prevalence of adenoma recurrence at SC1 in the whole cohort was $19.7 \%(n=81)$.

En bloc resection of polyps historically decreases adenoma recurrence risk on follow-up compared to piecemeal EMR [15]. In our cohort, there was a large difference in the rate of en bloc resection between the 2 groups $(39.2 \%$ [ $n=116$ ] with the 180 colonoscope versus $3.5 \%[n=4]$ with the 190 colonoscope; $P<.001)$. To avoid a bias in the results interpretation, en bloc resection cases were subsequently excluded $(n=120)$ from the final analysis. 
The baseline characteristics of the final study cohort are reported in $>$ Table 1. Our final cohort consisting of piecemeal only resections included 291 lesions; $61.9 \%(n=180)$ were resected with the 180 colonoscope and $38.1 \%(n=111)$ with the 190 colonoscope. Mean age was 67 years \pm 10 ( $57-77 \mathrm{y} / \mathrm{o})$, median lesion size was $30 \mathrm{~mm}$ ( $20-140 \mathrm{~mm})$, and median time to SC1 was 5 months (IQR $15-29$ weeks; - Fig. 2). The prevalence of adenoma recurrence at SC1 after piecemeal EMR was $24.1 \%$ $(n=70)$. There was no difference in the rate of RRA between the 180 and 190 scopes (23.3\% vs $25.2 \%$, respectively; $P=.82$ ). Except for age, prior treatment of polyp, initial histopathology, year of procedure, and use of APC, baseline characteristics were similar in both groups ( Table 2). During SC1, $59.1 \%$ (n $=172$ ) of the EMR scars were assessed with the 180 colonoscope and $40.5 \%(n=118)$ were assessed with the 190 colonoscope $(n=290)$. The remaining EMR scar was assessed by surgical resection secondary to RRA suspicious for malignancy. There was no evidence that the type of colonoscope used for surveillance had an impact in the rate of RRA $(P=.44)$.

IPW was applied to control for confounders, resulting in groups with balanced covariates ( $\downarrow$ Table 3 ). The OR estimate for effect of the 190 colonoscope on recurrence was 1.11 (95\% $\mathrm{Cl} 0.64-1.92 ; P=.71)$ before adjustment and $1.06(95 \% \mathrm{Cl}$ $0.60-1.86 ; P=.85)$ after IPW, suggesting no difference in adenoma recurrence based on the type of scope used for EMR ( Table 4).

\section{Risk factors for recurrence}

Adenoma size was documented as larger than $40 \mathrm{~mm}$ in $33 \%$ of the lesions $(n=96)$. The majority of the lesions were located in the right colon $(n=202,69.3 \%)$, with the ascending colon $(n=$ $97,33.3 \%)$ and cecum ( $n=63,21.6 \%)$ being the most common locations. HGD occurred in $6.9 \%$ of the lesions $(n=20)$ and IPB was reported in $10.7 \%$ of cases $(n=31)$.

Concomitant use of APC was documented in $26.1 \%$ of procedures $(n=76)$ and the frequency of lesions previously treated was $8.3 \%(n=24)$. On univariate logistic regression for all 411 lesions, risk factors for adenoma recurrence at SC1 were lesion size (OR 1.32 per $10 \mathrm{~mm}$; $95 \% \mathrm{Cl} 1.11-1.56$; $P=.002$ ) and complementary APC use (OR 3.31; $95 \% \mathrm{Cl} 1.93-5.67 ; P<.001)$. On univariate logistic regression for piecemeal only EMR $(n=291)$, APC use and a SERT score 1 - 4 were found as significant risk factors for adenoma recurrence ( $\triangleright$ Table 5 ).

Upon multivariate logistic regression with the 3 SERT variables for recurrence prediction (IPB, adenoma size, and HGD), none of the 3 were found as significant independent predictors for RRA at SC1 in our cohort ( $\triangleright$ Table 6 ).

\section{SERT score analysis}

From the cohort of 291 lesions with piecemeal resection, $59.8 \%$ $(n=174)$ were classified as SERT score 0 and $40.2 \%(n=117)$ had SERT scores of 1 to 4 . The overall recurrence at SC 1 was $24.1 \%$ $(n=70)$ and the recurrence at any follow-up colonoscopy was $25.4 \%(n=74)$. The mean time to recurrence or last follow-up was 63.5 weeks (IQR 19-81 weeks). The rates of recurrence for SERT scores 0 to 4 at SC1 were $20.1 \%, 27.3 \%, 27.1 \%$, $39.1 \%$, and $50 \%$, respectively ( $\downarrow$ Table 7 ). Lesions with SERT scores greater than 0 had a higher risk of recurrence when compared to those with a SERT score of 0 (OR 1.71; $95 \% \mathrm{Cl} 1.00-$ $2.92 ; P=.04)$.

Of 174 lesions classified as SERT score $0,79.9 \%(n=139)$ had no recurrence at SC1 and $20.1 \%(n=35)$ had recurrence at SC1. The accuracy of the SERT score ( 0 versus $>0$ ) for predicting recurrence was modest with a sensitivity of $50 \%$, a specificity of $63.1 \%$, a positive predictive value of $31.6 \%$, and a negative predictive value of $78.7 \%$. Thus, we estimate that $79 \%$ of lesions with SERT score 0 were correctly classified as low-risk and did not recur.

For the display of time to recurrence according to SERT score of the lesion, Kaplan-Meier survival plots were generated ( $\triangleright$ Fig. 3 and $>$ Fig.4). For lesions with SERT score 0, there was a $16.4 \%(n=26)$ recurrence at 6 months. The cumulative incidence of recurrence escalated to $23.3 \%(n=34)$ at 12 months and remained at $23.3 \%$ at 18 months. After 18 months, there was a minimal incidence of event rate at $24(25.4 \%, n=35)$ and 36 months $(27.5 \%, \mathrm{n}=36)$.

The cumulative incidence of lesions with SERT scores 1 to 4 was $21.9 \%(n=21)$ at 6 months, and consistently escalated at $12,18,24$, and 36 months $(37.6 \%, 43.6 \%, 47.1 \%$, and $51.5 \%$, respectively, log-rank test; $P=.009 ; \triangleright$ Table 8, $>$ Fig. 5).

\section{Discussion}

In our study, the use of second generation HD-NBI with NearFocus (190 colonoscopes) for EMR of large LSL did not affect the rate of adenoma recurrence at follow-up colonoscopy when compared to first generation HD-NBI series (180 colonoscopes). A SERT score of 0 was associated with lower recurrence rates, but was of modest accuracy at identifying patients who do not need intensive surveillance. A strength of this study was a large sample size of lesions resected throughout 6 years by a highly experienced group of endoscopists on a steady learning curve. The potential for outcomes to be affected by confounding variables as a consequence of the retrospective nature of the study was mitigated by the use of IPW. Nonetheless, as a tertiary referral center, we frequently treat complicated or previously manipulated lesions, potentially increasing the risk for adenoma recurrence and referral bias. Our recent implementation of snare tip soft coagulation for prevention of recurrence after EMR, might overestimate the benefits of 190 colonoscopes in reducing RRA rates. Results from a multicenter study have shown that thermal ablation (snare tip soft coagulation of margins) significantly reduced adenoma recurrence after EMR in SC1 [16]. Additionally, due to the retrospective nature of the study, it is uncertain if the endoscopists were indeed using the Near-Focus feature of 190 colonoscopes for every EMR.

Variable rates of RRA continue to challenge the effectiveness of colorectal EMR. Several studies have been conducted to find potential clinical and endoscopic predictors for recurrence [2, $3,7-10,19,20]$. A recent metanalysis of 30 articles and 3404 patients undergoing EMR reported an overall rate of recurrence of $13.1 \%$ [17]. Other studies found that the probability of clearance at first endoscopic resection attempt was affected by the 
- Table 1 Characteristics for 291 patients with piecemeal resections

\section{Characteristic}

Patients, No. (\%) ${ }^{1}$

Age

- Mean (SD)

$67(10.1)$

- Q1, Q3

60,75

- Range

$36-89$

Sex

\begin{tabular}{|l|r|}
\hline - Men & $132(45.4)$ \\
\hline - Women & $159(54.6)$ \\
\hline
\end{tabular}

Prior treatment

\begin{tabular}{|c|c|}
\hline - No & $267(91.8)$ \\
\hline - Yes & $24(8.2)$ \\
\hline
\end{tabular}

Polyp size, $\mathrm{mm}$

\begin{tabular}{|l|r|}
\hline . $20-39$ & $195(67.0)$ \\
\hline . $40+$ & $96(33.0)$ \\
\hline
\end{tabular}

EMR site

- Cecum $63(21.6)$

- Ileoceal valve

$16(5.5)$

- Ascending $97(33.3)$

- Hepatic flexure

$26(8.9)$

- Transverse $32(11.0)$

- Descending $13(4.5)$

- Sigmoid $16(5.5)$

- Anorectal $20(6.9)$

- Others $8(2.8)$

Initial pathology

\begin{tabular}{|l|c|}
\hline - HGD & $20(6.9)$ \\
\hline - TVA & $80(27.5)$ \\
\hline - SSA & $53(18.2)$ \\
\hline - TA & $122(41.9)$ \\
\hline - Adenocarcinoma & $8(2.7)$ \\
\hline - HP & $8(2.7)$ \\
\hline
\end{tabular}

HGD pathology

\begin{tabular}{l|c|}
\hline - Not HGD & $271(93.1)$ \\
\hline - HGD & $20(6.9)$ \\
\hline
\end{tabular}

\section{SERT score}

- N-Miss

0

- 0

$174(59.8)$

- 1

- 2

- 3

- 4

- 0
$22(7.6)$

$70(24.1)$

$23(7.9)$

$2(0.7)$

$117(40.2)$
- Table 1 (Continuation)

\begin{tabular}{|l|r|}
\hline Characteristic & Patients, \\
\hline Endoscopist & \\
\hline - 1 & $96(33.0)$ \\
\hline - 2 & $60(20.6)$ \\
\hline - 3 & $96(33.0)$ \\
\hline - 4 & $9(3.1)$ \\
\hline - 5 & $28(9.6)$ \\
\hline - 6 (Other) & $2(0.7)$ \\
\hline
\end{tabular}

Lifted lesion

- Adequate

$279(95.9)$

- Partial

$12(4.1)$

Intraprocedural bleeding

\begin{tabular}{|l|r|}
\hline - No & $260(89.3)$ \\
\hline - Yes & $31(10.7)$ \\
\hline
\end{tabular}

Prophylactic APC

\begin{tabular}{|l|r|}
\hline - No & $215(73.9)$ \\
\hline - Yes & $76(26.1)$ \\
\hline
\end{tabular}

Type of resection

- Piecemeal $291(100)$

- Enbloc $0(0)$

Scope used at SC1

\begin{tabular}{|l|c|}
\hline - 180 & $172(59.1)$ \\
\hline - 190 & $118(40.5)$ \\
\hline - Surgical specimen & $1(0.4)$ \\
\hline
\end{tabular}

Recurrence at first follow-up

\begin{tabular}{|l|r|}
\hline - No & $221(75.9)$ \\
\hline - Yes & $70(24.1)$ \\
\hline
\end{tabular}

Recurrence at any follow-up

\begin{tabular}{|l|r|}
\hline - No & $217(74.6)$ \\
\hline - Yes & $74(25.4)$ \\
\hline
\end{tabular}

Weeks to first follow-up
- Mean (SD)
$27.4(23.3)$
- Q1, Q3
$15,29.1$
- Range
$0-201$

Weeks to recurrence or last follow-up

\begin{tabular}{|l|c|}
\hline Mean (SD) & $63.5(66.6)$ \\
\hline Q1, Q3 & $18.9,80.8$ \\
\hline Range & $0-325$ \\
\hline
\end{tabular}

APC, argon plasma coagulation; EMR, endoscopic mucosal resection; HGD, high-grade dysplasia; HP, hyperplastic; N-Miss, number missing; Q1, first quartile; Q3, third quartile; SC1: first surveillance colonoscopy; SERT: Sydney EMR recurrence tool; SSA: sessile serrated adenoma; TA, tubular adenoma;

TVA, tubulovillous adenoma

1 Unless otherwise indicated 


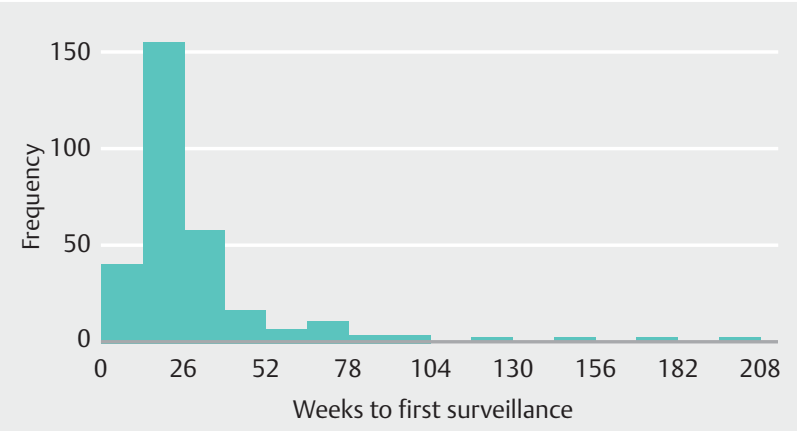

Fig. 2 Histogram showing the distribution of time to first surveillance colonoscopy.

- Table 2 Summary of piecemeal resections stratified by type of colonoscope used for EMR $(n=291)$.

\begin{tabular}{|c|c|c|c|}
\hline \multirow[t]{2}{*}{ Variable } & \multicolumn{2}{|c|}{ Colonoscope } & \multirow[t]{2}{*}{$P$ value } \\
\hline & $\begin{array}{l}180 \\
(n=180)^{1}\end{array}$ & $\begin{array}{l}190 \\
(n=111)^{1}\end{array}$ & \\
\hline Age & & & .03 \\
\hline - Mean (SD) & $68(10.1)$ & $65.3(9.85)$ & \\
\hline - Q1, Q3 & 62,75 & 59,72 & \\
\hline - Range & $40-88$ & $36-89$ & \\
\hline Sex & & & .84 \\
\hline - Male & $83(46.1)$ & $49(44.1)$ & \\
\hline - Female & 97 (53.9) & $62(55.9)$ & \\
\hline Prior treatment & & & .01 \\
\hline - No & $159(88.3)$ & $108(97.3)$ & \\
\hline - Yes & $21(11.7)$ & $3(2.7)$ & \\
\hline Polyp size, mm & & & .40 \\
\hline - N-Miss & 0 & 0 & \\
\hline - $20-29$ & $70(38.9)$ & $47(42.3)$ & \\
\hline - 30-39 & $46(25.6)$ & $32(28.8)$ & \\
\hline - $40-49$ & $34(18.9)$ & $15(13.5)$ & \\
\hline - $50-59$ & $16(8.9)$ & $11(9.9)$ & \\
\hline . $60-69$ & $7(3.9)$ & $2(1.8)$ & \\
\hline - 70-99 & $4(2.2)$ & $2(1.8)$ & \\
\hline - $100+$ & $3(1.7)$ & $2(1.8)$ & \\
\hline EMR site & & & .53 \\
\hline - Cecum & $43(23.9)$ & $20(18.0)$ & \\
\hline - Ileoceal valve & $11(6.1)$ & $5(4.5)$ & \\
\hline - Ascending & $51(28.3)$ & $46(41.4)$ & \\
\hline - Splenic flexure & $3(1.7)$ & $1(0.9)$ & \\
\hline - Hepatic flexure & $15(8.3)$ & $11(9.9)$ & \\
\hline - Transverse & $21(11.7)$ & $11(9.9)$ & \\
\hline
\end{tabular}

Table 2 (Continuation)

\begin{tabular}{|c|c|c|c|}
\hline \multirow[t]{2}{*}{ Variable } & \multicolumn{2}{|c|}{ Colonoscope } & \multirow[t]{2}{*}{$P$ value } \\
\hline & $\begin{array}{l}180 \\
(n=180)^{1}\end{array}$ & $\begin{array}{l}190 \\
(n=111)^{1}\end{array}$ & \\
\hline - Descending & $8(4.4)$ & $5(4.5)$ & \\
\hline - Sigmoid & $11(6.1)$ & $5(4.5)$ & \\
\hline - Anorectal & $15(8.3)$ & $5(4.5)$ & \\
\hline - Appendiceal orifice & $1(0.6)$ & $2(1.8)$ & \\
\hline - Colon multiple & $1(0.6)$ & $0(0.0)$ & \\
\hline Initial pathology & & & $<.001$ \\
\hline - TA with HGD & $4(2.2)$ & $3(2.7)$ & \\
\hline - TVA & $51(28.3)$ & $29(26.1)$ & \\
\hline - SSA & $23(12.8)$ & $38(34.2)$ & \\
\hline - TA & $90(50.0)$ & $32(28.8)$ & \\
\hline - TVA with HGD & $8(4.4)$ & $5(4.5)$ & \\
\hline - Adenocarcinoma & $4(2.2)$ & $4(3.6)$ & \\
\hline Paris Classification & & & .22 \\
\hline . Ip & $3(1.7)$ & $0(0.0)$ & \\
\hline - Is & $8(4.4)$ & $5(4.5)$ & \\
\hline - 0-IIa & $40(22.2)$ & $28(25.2)$ & \\
\hline - $0-I I b$ & $5(2.8)$ & $8(7.2)$ & \\
\hline . 0 -IIc & $2(1.1)$ & $0(0.0)$ & \\
\hline - IIc+IIa & $0(0.0)$ & $1(0.9)$ & \\
\hline - Unknown & $122(67.8)$ & $69(62.2)$ & \\
\hline Endoscopist & & & .08 \\
\hline - 1 & $57(31.7)$ & $39(35.1)$ & \\
\hline . 2 & $40(22.2)$ & $20(18.0)$ & \\
\hline .3 & $64(35.6)$ & $32(28.8)$ & \\
\hline . 4 & $2(1.1)$ & $7(6.3)$ & \\
\hline .5 & $15(8.3)$ & $13(11.7)$ & \\
\hline .6 & $2(1.1)$ & $0(0.0)$ & \\
\hline Year of procedure & & & $<.001$ \\
\hline - 2010 & $53(29.4)$ & $0(0.0)$ & \\
\hline - 2011 & $41(22.8)$ & $0(0.0)$ & \\
\hline - 2012 & $42(23.3)$ & $0(0.0)$ & \\
\hline - 2013 & $15(8.3)$ & $6(5.4)$ & \\
\hline - 2014 & $14(7.8)$ & $32(28.8)$ & \\
\hline . 2015 & $9(5.0)$ & $50(45.0)$ & \\
\hline - 2016 & $6(3.3)$ & $23(20.7)$ & \\
\hline Lifted lesion & & & .58 \\
\hline - Adequate & $174(96.7)$ & $105(94.6)$ & \\
\hline - Partial & $6(3.3)$ & $6(5.4)$ & \\
\hline Intraprocedural bleed & & & .90 \\
\hline
\end{tabular}


- Table 2 (Continuation)

\begin{tabular}{|c|c|c|c|}
\hline \multirow[t]{2}{*}{ Variable } & \multicolumn{2}{|l|}{ Colonoscope } & \multirow[t]{2}{*}{$P$ value } \\
\hline & $\begin{array}{l}180 \\
(n=180)^{1}\end{array}$ & $\begin{array}{l}190 \\
(n=111)^{1}\end{array}$ & \\
\hline - No & $160(88.9)$ & $100(90.1)$ & \\
\hline - Yes & $20(11.1)$ & $11(9.9)$ & \\
\hline Prophylactic APC & & & .04 \\
\hline - No & $141(78.3)$ & $74(66.7)$ & \\
\hline - Yes & $39(21.7)$ & $37(33.3)$ & \\
\hline Recurrence in EMR Scar & & & .98 \\
\hline - No & $138(76.7)$ & $83(74.8)$ & \\
\hline - Yes & $42(23.3)$ & $28(25.2)$ & \\
\hline Pathology of recurrence & & & .96 \\
\hline - No recurrence & $138(76.7)$ & $83(74.8)$ & \\
\hline - TVA & $11(6.1)$ & $6(5.4)$ & \\
\hline - SSA & $5(2.8)$ & $7(6.3)$ & \\
\hline - TA & $26(14.4)$ & $15(13.5)$ & \\
\hline Weeks to follow-up & & & .82 \\
\hline - N-Miss & 1 & 0 & \\
\hline - Mean (SD) & $27.9(27.6)$ & $27.2(14.4)$ & \\
\hline - Q1, Q3 & $14.1,27.6$ & $16.5,30.3$ & \\
\hline - Range & $6-201$ & $3.57-77.4$ & \\
\hline
\end{tabular}

APC, argon plasma coagulation; EMR, endoscopic mucosal resection; HGD, high-grade dysplasia; $\mathrm{N}$-Miss, number missing; Q1, first quartile; Q3, third quartile; SSA, sessile serrated adenoma; TA, tubular adenoma; TVA, tubulovillous adenoma

- Table 3 Summary of controls weighted to match cases (en bloc resection excluded; $n=291$ ).

\begin{tabular}{|c|c|c|}
\hline \multirow[t]{2}{*}{ Variable } & \multicolumn{2}{|l|}{ Colonoscope } \\
\hline & $180(n=180)^{1}$ & $190(n=111)^{1}$ \\
\hline \multicolumn{3}{|l|}{ Age } \\
\hline - Mean (SD) & $66.7(9.95)$ & $66.3(10.4)$ \\
\hline - Q1, Q3 & 60,75 & $59.5,75$ \\
\hline - Range & $40-88$ & $36-89$ \\
\hline \multicolumn{3}{|l|}{ Sex } \\
\hline - Men & $78.7(43.7)$ & $47(42.4)$ \\
\hline - Women & $101(56.3)$ & $64(57.6)$ \\
\hline \multicolumn{3}{|c|}{ Prior treatment } \\
\hline - No & $166(92.5)$ & $109(97.8)$ \\
\hline - Yes & $13.5(7.5)$ & $2.45(2.2)$ \\
\hline \multicolumn{3}{|l|}{ Polyp size, mm } \\
\hline - N-Miss & 0 & 0 \\
\hline . $20-29$ & $74.9(41.6)$ & $46.1(41.5)$ \\
\hline
\end{tabular}

- Table 3 (Continuation)

\begin{tabular}{|c|c|c|}
\hline \multirow[t]{2}{*}{ Variable } & \multicolumn{2}{|l|}{ Colonoscope } \\
\hline & $180(n=180)^{1}$ & $190(n=111)^{1}$ \\
\hline - 30-39 & $49.7(27.6)$ & $31.8(28.6)$ \\
\hline - $40-49$ & $28.9(16.1)$ & $16.4(14.7)$ \\
\hline - 50-59 & $15.5(8.6)$ & $9.53(8.6)$ \\
\hline - $60-69$ & $5.33(2.96)$ & $4.42(3.98)$ \\
\hline - 70-99 & $3.09(1.8)$ & $1.52(1.4)$ \\
\hline - $100+$ & $2.58(1.4)$ & $1.34(1.2)$ \\
\hline \multicolumn{3}{|l|}{ EMR site } \\
\hline - Cecum & $36.3(20.2)$ & $23.2(20.9)$ \\
\hline - Ileoceal valve & $10.6(5.9)$ & $4.69(4.2)$ \\
\hline - Ascending & $65.9(36.6)$ & $37.6(33.9)$ \\
\hline - Splenic flexure & $2.05(1.1)$ & $0.651(0.6)$ \\
\hline - Hepatic flexure & $15.6(8.7)$ & $10.8(9.8)$ \\
\hline - Transverse & $18.8(10.5)$ & $13(11.8)$ \\
\hline - Descending & $7.71(4.3)$ & $5.77(5.2)$ \\
\hline - Sigmoid & $9.48(5.3)$ & $7.83(7.1)$ \\
\hline - Anorectal & $11.6(6.4)$ & $6.15(5.5)$ \\
\hline $\begin{array}{l}\text { - Appendiceal ori- } \\
\text { fice }\end{array}$ & $1.18(0.7)$ & $1.18(1.1)$ \\
\hline - Colon multiple & $0.603(0.3)$ & $0(0)$ \\
\hline \multicolumn{3}{|l|}{ Initial pathology } \\
\hline - TA with HGD & $4.62(2.6)$ & 4.27 (3.9) \\
\hline - TVA & $48.6(27)$ & $34.3(30.9)$ \\
\hline - SSA & $37.1(20.6)$ & $25(22.5)$ \\
\hline - TA & $79.9(44.4)$ & $41.8(37.6)$ \\
\hline - TVA with HGD & $6.5(3.6)$ & $3.39(3.1)$ \\
\hline - Adenocarcinoma & $3.24(1.8)$ & $2.29(2.1)$ \\
\hline \multicolumn{3}{|l|}{ Paris classification } \\
\hline - Ip & $1.81(1.0)$ & $0(0)$ \\
\hline - Is & $9.93(5.5)$ & $6.29(5.7)$ \\
\hline - 0-lla & $37.8(21)$ & $23.3(21)$ \\
\hline - $0-I I b$ & $6.4(3.6)$ & $5.32(4.8)$ \\
\hline . 0 -IIc & $1.21(0.7)$ & $0(0)$ \\
\hline - IIc+lla & $0(0)$ & $0.427(0.4)$ \\
\hline - Unknown & $123(68.2)$ & $75.7(68.2)$ \\
\hline \multicolumn{3}{|l|}{ Endoscopist } \\
\hline - 1 & $60.8(33.8)$ & $35.7(32.1)$ \\
\hline .2 & $34.7(19.3)$ & $21(18.9)$ \\
\hline .3 & $56.9(31.6)$ & $39.1(35.3)$ \\
\hline - 4 & $9.51(5.3)$ & $4(3.6)$ \\
\hline . 5 & $16.9(9.4)$ & $11.2(10.1)$ \\
\hline . 6 & $1.21(0.7)$ & $0(0)$ \\
\hline
\end{tabular}


- Table 3 (Continuation)

\begin{tabular}{|c|c|c|}
\hline \multirow[t]{2}{*}{ Variable } & \multicolumn{2}{|l|}{ Colonoscope } \\
\hline & $180(n=180)^{1}$ & $190(n=111)^{1}$ \\
\hline \multicolumn{3}{|l|}{ Year of procedure } \\
\hline . 2010 & $47.4(26.3)$ & $0(0)$ \\
\hline . 2011 & $34.6(19.2)$ & $0(0)$ \\
\hline . 2012 & $39.5(22)$ & $0(0)$ \\
\hline . 2013 & $17.6(9.8)$ & $8.85(7.97)$ \\
\hline - 2014 & $16.5(9.2)$ & $33.9(30.5)$ \\
\hline . 2015 & $9.96(5.5)$ & $47.2(42.6)$ \\
\hline . 2016 & $14.4(7.99)$ & $21(18.9)$ \\
\hline \multicolumn{3}{|l|}{ Lifted lesion } \\
\hline - Adequate & $173(96)$ & $106(95.4)$ \\
\hline - Partial & $7.12(3.95)$ & $5.06(4.6)$ \\
\hline \multicolumn{3}{|c|}{ Intraprocedural bleeding } \\
\hline - No & $161(89.4)$ & $100(90.5)$ \\
\hline - Yes & $19.1(10.6)$ & $10.6(9.5)$ \\
\hline \multicolumn{3}{|l|}{ Prophylactic APC } \\
\hline - No & $133(73.8)$ & $78.5(70.7)$ \\
\hline - Yes & $47.1(26.2)$ & $32.5(29.3)$ \\
\hline \multicolumn{3}{|c|}{ Recurrence in EMR scar } \\
\hline - No & $140(78)$ & $85.4(77)$ \\
\hline - Yes & $39.7(22)$ & $25.6(23)$ \\
\hline \multicolumn{3}{|c|}{ Pathology of recurrence } \\
\hline - No recurrence & $140(78)$ & $84.2(75.8)$ \\
\hline - TVA & $9.78(5.4)$ & $5.73(5.2)$ \\
\hline - SSA & $7.51(5.7)$ & $4.34(3.9)$ \\
\hline - TA & $22.4(12.4)$ & $15.5(14)$ \\
\hline \multicolumn{3}{|l|}{ Weeks to follow-up } \\
\hline - N-Miss & 0.903 & 0 \\
\hline - Mean (SD) & $27.5(25.5)$ & $27.4(14.8)$ \\
\hline - Q1, Q3 & $14.6,28.9$ & $16.5,30.7$ \\
\hline - Range & $6-201$ & $3.57-77.4$ \\
\hline \multicolumn{3}{|c|}{$\begin{array}{l}\text { APC, argon plasma coagulation; EMR, endoscopic mucosal resection; HGD, } \\
\text { high-grade dysplasia; N-Miss, number missing; Q1, first quartile; Q3, third } \\
\text { quartile; SSA, sessile serrated adenoma; TA, tubular adenoma; TVA, tubulo- } \\
\text { villous adenoma } \\
{ }^{1} \text { No. (\%) unless otherwise indicated }\end{array}$} \\
\hline
\end{tabular}

lesion complexity and there was a significant drop in complete cure rate at first attempt for lesions larger than $60 \mathrm{~mm}$ and for very complex or defiant adenomas with difficult access $[18,19]$. Similarly, we found in our study a clear association between the rate of RRA and polyp size, supporting what literature has consistently shown $[10,11,19,20]$. A retrospective study identified polyp size, piecemeal resection, concomitant use of APC, ex- tension to lateral margins, and presence of HGD in histopathology as risk factors for RRA [19].

Large LSL (> $20 \mathrm{~mm}$ ) are generally resected with a piecemeal method. A systematic review and meta-analysis demonstrated that local recurrence after EMR occurs in $3 \%$ of cases in which the lesion is removed en bloc and in $20 \%$ of cases in which the lesion is removed with a piecemeal technique $[17,21]$. Current ASGE guidelines recommend that if a piecemeal resection is performed on a large adenoma (>15 mm), the patient should have a subsequent colonoscopy in 6 to 12 months to evaluate for local recurrence [22], which entails a financial impact in the healthcare system and may be distressing for the patient.

In a retrospective study by Woodward et al [20], additional procedures were needed to achieve complete resection in more than 1 of 10 colonic EMRs and residual neoplasia occurred more often if the lesion was resected in pieces. Furthermore, the use of complementary APC for complete resection has consistently shown higher rates of RRA (47\% [19] and 33\% [10]). Our study corroborates these findings showing a substantial increase of recurrence in patients who received APC as part of the therapy. Typically, the APC settings used in our center included0.8 liters/minute; Erbe, Tubigen, Germany, with 20 watts current flow and mostly used at the right colon. Interestingly, a recent study by Holmes et al [23] reported that avulsion is superior to APC for the treatment of residual visible neoplasia, decreasing the recurrence rate without increasing the procedure risks. Since we started using hot avulsion and snare tip soft coagulation in our EMR practice only after later 2016; there were only few cases that were not included in the final analysis to avoid bias.

Resections of fibrotic and scarred polyps are technically difficult as it possess the risk of perforation [24]. However, APC and cold avulsion technique can be used as a salvage approach to achieve complete removal of partially resected, non-lifting, and fibrotic polyps after piecemeal EMR [25]. There are few novel techniques for complete removal of large laterally spreading colorectal lesions such as hybrid techniques in which lateral margins are freed with circumferential SM incision and facilitated by submucosal elevation followed by snare resection [26]. In addition, Hybrid- knife is a novel device in which submucosal dissection is combined with injection of fluid concurrently [27]. This device has increased the efficiency of ESD on western endoscopists.

The ASGE recommends against EMR for non-lifting lesions or lesions classified as Paris II-c/III. However, non-lifting lesions that were manipulated (biopsy or attempted EMR) before referral for resection are usually amenable to EMR [22]. Our institution is a tertiary referral center and a large number of LSL have been previously manipulated, creating fibrosis and tissue scarring and making a complete resection theoretically more challenging. However, we did not find a strong association between prior treatment of the lesion and a higher rate of RRA in our cohort. A study limitation is that prior biopsy or treatment of the lesion in another facility could have been unreported. Moreover, these lesions are often treated with supplemental APC, which limits the interpretation of the results. 
- Table 4 Effect of the Olympus 190 colonoscope on adenoma recurrence at different stages of adjustment.

\begin{tabular}{|l|l|l|l|l|}
\hline Model & Odds ratio & Lower $95 \% \mathbf{C l}$ & Upper $\mathbf{9 5 \%} \mathbf{C l}$ \\
\hline Unadjusted-all patients (en bloc and piecemeal) & 1.58 & 0.94 & 2.65 \\
\hline Unadjusted-piecemeal resection only & 1.11 & 0.64 & 1.92 \\
\hline IPW adjustment-piecemeal resection & 1.06 & 0.60 & 1.86 & .08 \\
\hline CI, confidence interval; IPW, inverse probability weighting & & & .71 \\
\hline
\end{tabular}

- Table 5 Unadjusted measures of association of different variables with probability of recurrence

\begin{tabular}{|c|c|c|c|c|c|}
\hline Variable & OR & Lower $95 \% \mathrm{Cl}$ & Upper $95 \%$ Cl & P Value & Concordance \\
\hline SERT 1 & 1.39 & 0.471 & 3.64 & .52 & 0.575 \\
\hline SERT 2 & 1.59 & 0.839 & 2.96 & .15 & . \\
\hline SERT 3 & 2.38 & 0.927 & 5.87 & .06 & . \\
\hline SERT 4 & 3.7 & 0.144 & 95.1 & .36 & . \\
\hline SERT $>0$ & 1.71 & 1 & 2.92 & .05 & 0.566 \\
\hline Size $40 \mathrm{~mm}+$ & 1.69 & 0.973 & 2.91 & .06 & 0.56 \\
\hline Pathology HGD & 0.976 & 0.308 & 2.62 & .96 & 0.501 \\
\hline IPB & 1.72 & 0.759 & 3.73 & .18 & 0.528 \\
\hline Age & 0.835 & 0.641 & 1.08 & .18 & 0.554 \\
\hline Sex: female & 0.969 & 0.571 & 1.65 & .91 & 0.504 \\
\hline Prior treatment & 0.394 & 0.091 & 1.19 & .14 & 0.528 \\
\hline Ileocecal valve & 0.678 & 0.142 & 2.44 & .58 & 0.568 \\
\hline Ascending colon & 0.812 & 0.386 & 1.73 & .58 & . \\
\hline Hepatic flexure & 1.08 & 0.367 & 2.98 & .88 & . \\
\hline Transverse colon & 0.979 & 0.354 & 2.57 & .97 & . \\
\hline Descending colon & 1.31 & 0.319 & 4.63 & .69 & . \\
\hline Sigmoid colon & 0.979 & 0.247 & 3.28 & .97 & . \\
\hline Anorectal & 1.26 & 0.392 & 3.74 & .69 & . \\
\hline Others & 4.9 & 1.08 & 26.1 & .04 & . \\
\hline Complementary APC & 2.53 & 1.43 & 4.47 & .001 & 0.597 \\
\hline SC1 with 190 scope & 1.25 & 0.71 & 2.18 & .44 & - \\
\hline
\end{tabular}

APC, argon plasma coagulation; HGD, high-grade dysplasia; IPB, intraprocedural bleeding; OR, odds ratio; TA, tubular adenoma; SC1, first surveillance colonoscopy; SERT, Sydney endoscopic mucosal resection recurrence tool; SSA, sessile serrated adenoma; TA, tubular adenoma; TVA, tubulovillous adenoma.

- Table 6 Multivariate model based on SERT variables.

\begin{tabular}{|l|l|l|l|l|l|}
\hline Variable & SE & OR & Lower 95\% Cl & Upper $95 \%$ Cl & P value \\
\hline Size $40 \mathrm{~mm}^{+}$ & 0.288 & 1.695 & 0.963 & 2.983 & .07 \\
\hline HGD & 0.555 & 0.771 & 0.260 & 2.287 & .64 \\
\hline IPB & 0.407 & 1.607 & 0.724 & 3.566 & .24 \\
\hline
\end{tabular}

HGD, high-grade dysplasia; IPB: intraprocedural bleeding; OR, odds ratio; SE, standard error; SERT, Sydney endoscopic mucosal resection recurrence tool 
- Table 7 Recurrence rates By SERT score (0 - 4).

\begin{tabular}{|c|c|c|c|c|c|c|}
\hline Event & $0(n=174)^{1}$ & $1(n=22)^{1}$ & $2(n=70)^{1}$ & $3(n=23)^{1}$ & $4(n=2)^{1}$ & $P$ value \\
\hline Recurrence at any follow-up & & & & & & .26 \\
\hline - No & $137(78.7)$ & $16(72.7)$ & $49(70)$ & $14(60.9)$ & $1(50)$ & \\
\hline - Yes & $37(21.3)$ & $6(27.3)$ & $21(30)$ & $9(39.1)$ & $1(50)$ & \\
\hline Recurrence at first follow-up & & & & & & .23 \\
\hline - No & $139(79.9)$ & $16(72.7)$ & $51(72.9)$ & $14(60.9)$ & $1(50)$ & \\
\hline - Yes & $35(20.1)$ & $6(27.3)$ & $19(27.1)$ & $9(39.1)$ & $1(50)$ & \\
\hline
\end{tabular}

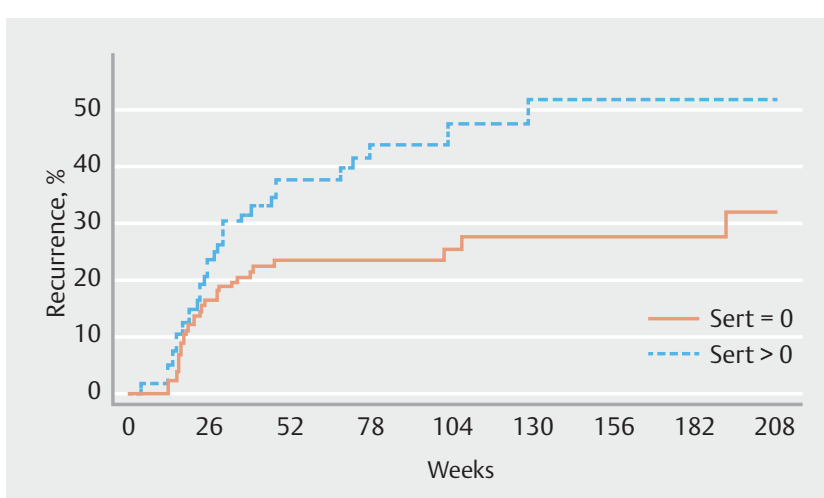

- Fig. 3 Kaplan-Meier plot for time to recurrence of lesions with SERT score 0 (continuous line) and $>0$ (dashed line).

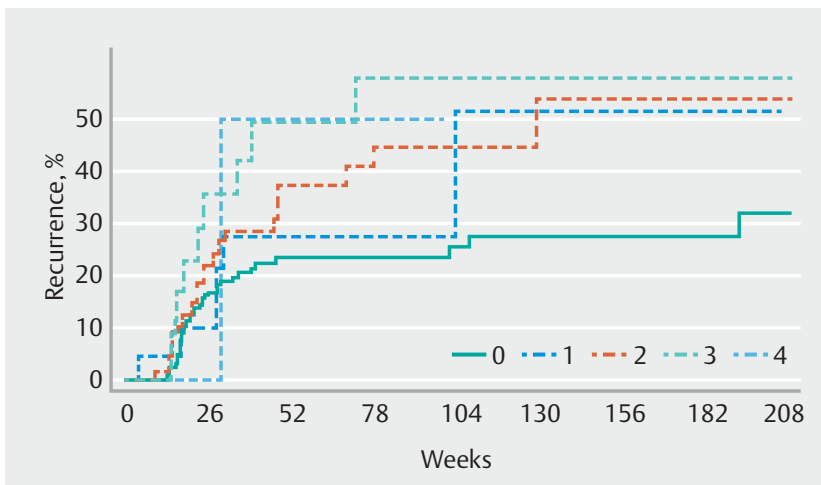

- Fig. 4 Kaplan-Meier plot for time to recurrence of lesions with SERT score 0 (green continuous line) and 1, 2, 3, and 4 (dashed lines).

IPB rates during EMR of colorectal lesions larger than $20 \mathrm{~mm}$ are reported to be between $11 \%$ and $22 \%$. IPB obscures the endoscopic view and shifts the endoscopist's focus away from the resection, compromising its efficacy $[28,29]$.

We speculated that improved imaging capabilities may lead to better index EMR visualization and effectiveness. This was based on recent studies regarding the 190 , second generation HD-NBI colonoscopes. A prospective study by Szura et al [30] found that using a 2-stage optical system (DF and HD-NBI) increased diagnostic accuracy for differentiating colorectal polyps with neoplastic potential (Kudo III to V). On the other hand, our own prospective study found "no difference in the accuracy of polyp histology prediction, adenoma detection, or surveillance interval prediction when comparing the 180 series with the dual focus 190 series colonoscopes" [5]. It remains possible that there are small incremental improvements with each generation of endoscopes, but these are too small to demonstrate statistically significant differences. We were unable to compare EMR done with prior, standard definition colonoscopes (Olympus 160 series or earlier) due to the small number of patients who underwent colonoscopy with this series and potential learning curve variations prior to 2010 .

Preliminary data from an ongoing, prospective, double-blind trial by Kandel et al [31], have indicated a high diagnostic accuracy with $\mathrm{HD}-\mathrm{NBI}$ near-focus system for optical detection of residual neoplasia in both real-time and offline evaluation. One hundred seven patients with 111 scar sites have been evaluated; sensitivity and negative predictive value are both $100 \%$ for HD-NBI near-focus with high confidence in real-time. Therefore, these advanced imaging modalities may improve realtime decision making for surveillance after colorectal EMR, particularly biopsy avoidance.

The recent development of SERT has demonstrated that RRA after EMR is predictable and stratifiable. In our cohort, SERT was capable of separating lesions with low risk for recurrence (SERT score 0 ) from lesions with higher recurrence rates (SERT score $>0$ ). Cumulative incidence for each group of lesions demonstrated that lesions with SERT score 0 still need to be surveilled at 6 and 18 months after index EMR as RRA were found in $6.9 \%$ $(n=8)$ of cases in this time frame. Lesions with SERT score 0 that were negative at 18 months remained negative on subsequent surveillance, displaying a plateau in Kaplan-Meier curves. Our data suggests that lesions with SERT score 0 that are negative for recurrence at second SC could potentially undergo routine SC. Conversely, Lesions with SERT scores 1 to 4 exhibited a higher cumulative incidence of histologically determined recurrence over time, suggesting a need for continued surveillance protocol for these patients. It is important to acknowledge that in multivariate analysis, none of the SERT model variables were significantly associated with recurrence. This could be 
- Table 8 Cumulative incidence of recurrence at SC1 by SERT score in 291 Lesions.

\begin{tabular}{|l|l|l|l|l|}
\hline Months & No. events [SERT = 0] & Event rate, \% [SERT = 0] & No. events [SERT > $\mathbf{~ ] ~}$ & Event rate, \% [SERT > 0] \\
\hline 6 & 26 & 16.4 & 21 & 21.9 \\
\hline 12 & 34 & 23.3 & 32 & 37.6 \\
\hline 18 & 34 & 23.3 & 35 & 43.6 \\
\hline 24 & 35 & 25.4 & 36 & 47.1 \\
\hline 30 & 36 & 27.5 & 37 & 51.5 \\
\hline 36 & 36 & 27.5 & 37 & 51.5 \\
\hline SC1, first surveillance colonoscopy; SERT, Sydney endoscopic mucosal resection recurrence tool
\end{tabular}

\begin{tabular}{|c|c|c|}
\hline \multicolumn{2}{|c|}{$n=291$} & \multirow[b]{4}{*}{$0-3$ months } \\
\hline$\downarrow$ & $\downarrow$ & \\
\hline $\mathrm{SERT}=0 \quad \mathrm{n}=174$ & SERT $>0 \quad n=117$ & \\
\hline 2 events ( $1.1 \%) \quad 5$ censored & 5 events $(4.3 \%) \quad 8$ censored & \\
\hline$n=167$ & $n=104$ & \\
\hline 24 events (14.4\%) 30 censored & 16 events (15.4\%) 26 censored & $3-6$ months \\
\hline$n=113$ & $n=62$ & \\
\hline 8 events $(7.1 \%) \quad 31$ censored & 11 events (17.7 \%) 13 censored & $6-12$ months \\
\hline$n=74$ & $n=38$ & \\
\hline 3 events (4.19\%) & 5 events (13.2\%) & $>12$ months \\
\hline
\end{tabular}

Fig. 5 Flowchart showing SERT 0 and SERT >0 recurrences in different time windows.

secondary to a smaller sample size and wider Cls compared to the study by Tate et al [12]. Interpretation of Kaplan-Meier curves was limited by the time at which the patients presented for SC. While the median time to SC1 was 5 months ( $\mathbf{F i g . 2 ) , ~}$ there were some outliers who presented later, in whom recurrence could have been previously found if surveilled between 4 to 6 months. Further validation in a prospective study is required for clinical application of the SERT model in our patient population.

\section{Conclusion}

This is a retrospective study and results show that the 190 , second generation colonoscopes with HD-NBI imaging and near focus magnification don't have an impact in the rate of adenoma recurrence at follow-up colonoscopy. These results need to be further substantiated with prospective studies, ideally randomizing the participants to EMR with either HD-NBI and DF magnification or HD-NBI alone. A key aspect for accuracy in the interpretation of the results is the endoscopist's continuous education for better implementation of novel technologies in their practice. Evidence suggests that adenoma recurrence might be associated with adenoma size, HGD, prior treatment of the lesion, IPB, complementary APC, and endoscopist's expertise [32].

\section{Competing interests}

Dr. Wallace reports consulting income from Olympus and grant support from Boston Scientific, Olympus, Medtronic and Cosmo pharmaceuticals.

\section{References}

[1] ASGE TechnicalCommittee. High-definition and high-magnification endoscopes. Gastrointest Endosc 2014; 80: 919-927 
[2] Moss A, Bourke M], Williams S] et al. Endoscopic mucosal resection outcomes and prediction of submucosal cancer from advanced colonic mucosal neoplasia. Gastroenterol 2011; 140: 1909-1918

[3] Desomer L, Tutticci N, Tate DJ et al. 1002: A standardized imaging protocol is accurate in detecting recurrence after endoscopic mucosal resection. Gastrointest Endosc 2016; 83: AB190

[4] Leung WK, Lo OS, Liu KS et al. Detection of colorectal adenoma by narrow band imaging (HQ190) vs. high-definition white light colonoscopy: a randomized controlled trial. Am J Gastroenterol 2014; 109: $855-863$

[5] Wallace MB, Crook JE, Coe S et al. Accuracy of in vivo colorectal polyp discrimination by using dual-focus high-definition narrow-band imaging colonoscopy. Gastrointest Endosc 2014; 80: 1072-1087

[6] Cohen J. The benefit of narrow-band imaging after EMR of laterally spreading lesions. Gastrointest Endosc 2017; 85: 527-529

[7] Briedigkeit A, Sultanie O, Sido B et al. Endoscopic mucosal resection of colorectal adenomas $>20 \mathrm{~mm}$ : Risk factors for recurrence. World J Gastrointest Endosc 2016; 8: 276-281

[8] Jang ES, Kim JW, Jung YJ et al. Clinical and endoscopic predictors of colorectal adenoma recurrence after colon polypectomy. Turk J Gastroenterol 2013; 24: 476-482

[9] Jang HW, Park S], Hong SP et al. Risk factors for recurrent high-risk polyps after the removal of high-risk polyps at initial colonoscopy. Yonsei Med J 2015; 56: 1559-1565

[10] Margagnoni G, Angeletti S, D'Ambra G et al. Outcome and risk of recurrence for endoscopic resection of colonic superficial neoplastic lesions over $2 \mathrm{~cm}$ in diameter. Dig Liver Dis 2016; 48: 399-403

[11] Zhan T, Hielscher T, Hahn F et al. Risk factors for local recurrence of large, flat colorectal polyps after endoscopic mucosal resection. Digestion 2016; 93: 311-317

[12] Tate DJ, Desomer L, Klein A et al. Adenoma recurrence after piecemeal colonic EMR is predictable: the Sydney EMR recurrence tool. Gastrointest Endosc 2017; 85: 647 - 656 e646

[13] The Paris endoscopic classification of superficial neoplastic lesions: esophagus, stomach, and colon: November 30 to December 1, 2002. Gastrointest Endosc 2003; 58: S3-43

[14] Karita M, Tada M, Okita K et al. Endoscopic therapy for early colon cancer: the strip biopsy resection technique. Gastrointest Endosc 1991; 37: $128-132$

[15] Knabe M, Pohl J, Gerges C et al. Standardized long-term follow-up after endoscopic resection of large, nonpedunculated colorectal lesions: a prospective two-center study. Am J Gastroenterol 2014; 109: $183-189$

[16] Klein A, Jayasekeran V, Hourigan LF et al. 812b A multi-center randomized control trial of thermal ablation of the margin of the post endoscopic mucosal resection (EMR) mucosal defect in the prevention of adenoma recurrence following EMR: Preliminary results from the "SCAR" study. Gastroenterol 2016; 150: S1266-S1267

[17] Ortiz AM, Bhargavi P, Zuckerman M] et al. Endoscopic mucosal resection recurrence rate for colorectal lesions. South Med J 2014; 107: $615-621$
[18] Longcroft-Wheaton G, Duku M, Mead R et al. Risk stratification system for evaluation of complex polyps can predict outcomes of endoscopic mucosal resection. Dis Colon Rectum 2013; 56: 960 - 966

[19] Buchner AM, Guarner-Argente C, Gisberg GG. Outcomes of EMR of defiant colorectal lesions directed to an endoscopy referral center. Gastrointest Endosc 2012; 76: 255 - 263

[20] Woodward TA, Heckman MG, Cleveland P et al. Predictors of complete endoscopic mucosal resection of flat and depressed gastrointestinal neoplasia of the colon. Am J Gastroenterol 2012; 107: 650 654

[21] Belderbos TDG, Leenders M, Moons LMG et al. Local recurrence after endoscopic mucosal resection of nonpedunculated colorectal lesions: systematic review and meta-analysis. Endoscopy 2014; 46: 388-402

[22] Hwang JH, Konda V, Abu DayyehBK et al. Endoscopic mucosal resection. Gastrointest Endosc 2015; 82: 215 - 226

[23] Holmes I, Kim HG, Yang DH et al. Avulsion is superior to argon plasma coagulation for treatment of visible residual neoplasia during EMR of colorectal polyps (with videos). Gastrointest Endosc 2016; 84: 822 829

[24] Tsiamoulos ZP, Bourikas LA, Saunders BP. Endoscopic mucosal ablation: a new argon plasma coagulation/injection technique to assist complete resection of recurrent, fibrotic colon polyps (with video). Gastrointest Endosc 2012; 75: 400 - 404

[25] Tsiamoulos ZP, Rameshshanker R, Gupta S et al. Augmented endoscopic resection for fibrotic or recurrent colonic polyps using an ablation and cold avulsion technique. Endoscopy 2016; 48: E248 -E249

[26] Holt BA, Bourke MJ. Wide field endoscopic resection for advanced colonic mucosal neoplasia: Current status and future directions. Clin Gastroenterol Hepatol 2012; 10: 969-979

[27] Lingenfelder T, Fischer K, Sold MG et al. Combination of water-jet dissection and needle-knife as a hybrid knife simplifies endoscopic submucosal dissection. Surg Endosc 2009; 23: 1531-1535

[28] Fahrtash-Bahin F, Holt BA, Jayasekeran V et al. Snare tip soft coagulation achieves effective and safe endoscopic hemostasis during widefield endoscopic resection of large colonic lesions (with videos). Gastrointest Endosc 2013; 78: 158 -163.e151

[29] Burgess NG, Metz A], Williams S] et al. Risk factors for intraprocedural and clinically significant delayed bleeding after wide-field endoscopic mucosal resection of large colonic lesions. Clin Gastroenterol Hepatol 2014; 12: 651-661.e653

[30] Szura M, Pasternak A, Bucki K et al. Two-stage optical system for colorectal polyp assessments. Surg Endosc 2016; 30: 204-214

[31] Kandel P, Brand EC, Chen WC et al. 690 diagnostic accuracy of optical detection of colorectal neoplasia after endoscopic mucosal resection: Prospective double blind comparison of high definition white light, narrow band imaging and near focus. Gastrointest Endosc 2017; 85: AB101-AB102

[32] Bhurwal A, Bartel M], Heckman MG et al. Endoscopic mucosal resection: learning curve for large nonpolypoid colorectal neoplasia. Gastrointest Endosc 2016; 84: 959 - 968 e957 